\title{
Palmprint Verification Using Multi-scale Gradient Orientation Maps
}

\author{
Min-Ki Kim* \\ Research Institute of Computer and Information Communication, \\ Department of Computer Science Education, Gyeongsang National University, Jinju 660-701, Korea
}

(Received December 30, 2010 : revised February 23, 2011 : accepted February 23, 2011)

\begin{abstract}
This paper proposes a new approach to palmprint verification based on the gradient, in which a palm image is considered to be a three-dimensional terrain. Principal lines and wrinkles make deep and shallow valleys on a palm landscape. Then the steepest slope direction in each local area is first computed using the Kirsch operator, after which an orientation map is created that represents the dominant slope direction of each pixel. In this study, three orientation maps were made with different scales to represent local and global gradient information. Next, feature matching based on pixel-unit comparison was performed. The experimental results showed that the proposed method is superior to several state-of-the-art methods. In addition, the verification could be greatly improved by fusing orientation maps with different scales.
\end{abstract}

Keywords: Palmprint verification, Slope direction, Kirsch operator, Orientation map

OCIS codes : (070.5010) Pattern recognition; (100.2000) Digital image processing; (070.4560) Data processing by optical means

\section{INTRODUCTION}

With the rapid progress of electronics and Internet commerce, personal identification is becoming increasingly important. Biometrics technology is considered one of the safest and most efficient ways to identify individuals or discriminate between an authorized person and an impostor. Biometrics comprises methods of uniquely recognizing persons based on their intrinsic physical (e.g., fingerprint, finger geometry, face, iris, etc.) or behavioral (e.g., voice, signature, gate, etc.) characteristics [1-3]. Among these traits, the palmprint has attracted many researchers' attention in the last 10 years. The palmprint, the large inner surface of the hand, contains many unique features such as principal lines, wrinkles, ridges, minutiae points, singular points, and texture. Earlier studies focused on high-resolution (at least $400 \mathrm{dpi}$ ) palmprint images for some applications such as law enforcement. Nowadays, most studies focus on low-resolution palmprint images for civil and commercial applications.

There are many approaches to palmprint recognition. Line-based approaches concentrate on extracting palm lines such as principal lines and wrinkles [4-8]. The extracted lines are usually encoded based on their orientation and magnitude. Appearance-based approaches use holistic appearance features such as principal component analysis [9, 10], the Fisher linear discriminant [11], and independent component analysis [12]. Statistical approaches transform an input image into another domain and divide the transformed image into small regions. Then statistical features are extracted from each small region $[13,14]$. According to the results of these studies, line-based approaches are deemed to produce the most promising results. In particular, some studies [5, $7,8]$ based on line orientation information are considered the state-of-the-art methods. Kong and Zhang [5] used six 2D Gabor filters with different directions, and extracted the dominant orientation information using the winner-takes-all rule. $\mathrm{Wu}$ et al. [7] devised four directional templates to define the orientation of each pixel. Jia et al. [8] devised another six directional templates based on modified finite Radon transformation.

In line-based methods, it is important to classify points as located on a palm line or not. This classification poses a challenge. Kong and Zhang [5] assumed that each point on a palmprint image is located on a palm line. Under this assumption, the orientation of each point is extracted. This approach is simple and has shown promising results. Thus, the following researches [7, 8] used the same approach. This approach has some problems, though. The line structures

\footnotetext{
*Corresponding author: mkkim@gnu.ac.kr

Color versions of one or more of the figures in this paper are available online.
} 
in a palmprint image are complex, and multiple lines may intersect in some regions. Although the orientation information of the pixels that are on palm lines is robust to variations in lighting conditions, the orientation information of the other pixels could be vulnerable to changes in illumination. That is, the orientation information of a point that is not located on a palm line is more fragile than that of a point on a palm line. Furthermore, the majority of the points are not located on a palm line. To solve this problem, this paper proposes a new viewpoint. When a palmprint image is hypothetically considered a $3 \mathrm{D}$ terrain, the principal lines and wrinkles become deep and shallow valleys on a palm landscape. All the line-based methods have tried to detect the orientation of valleys, but this study focuses on the slope caused by deep and shallow valleys. The main goal of this study is to detect the orientation of the steepest slope in a local area.

The rest of this paper is organized as follows. Section 2 presents a preprocessing method of extracting a region of interest (ROI) in a palmprint image and proposes a new feature that represents the dominant slope orientation of each pixel. Section 3 presents the feature-coding method by which a gradient orientation map is created, and describes a feature-matching method based on pixel-unit comparison. In Section 4, the experimental results and analysis are provided, and finally, the conclusions are described in Section 5 .

\section{EXTRACTION OF PALMPRINT FEATURES}

\subsection{Palmprint Preprocessing}

When palmprint images are captured, some variations may occur such as in translation and rotation. Hence, palmprint images should be aligned in position and orientation before the feature extraction step $[15,16]$. The central part of a palm is a region of interest (ROI), from which a palmprint feature is extracted. The five main steps in cropping the

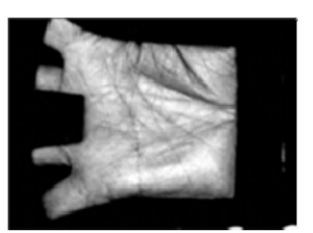

(a)

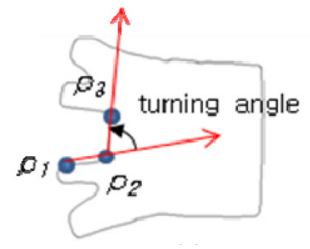

(e)

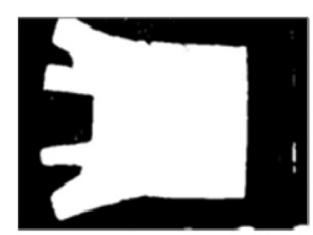

(b)

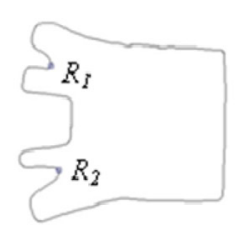

(f)

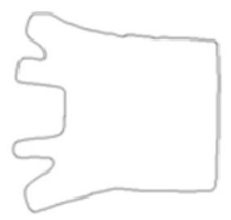

(c)

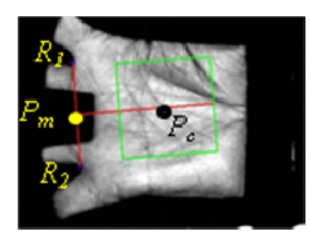

(g)

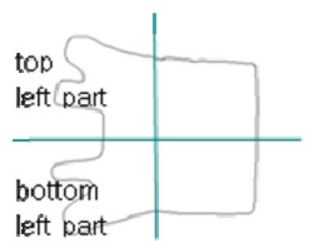

(d)

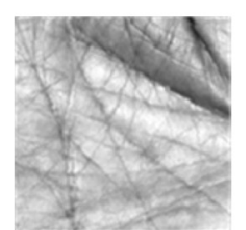

(h)

FIG. 1. The main steps in the preprocessing: (a) original image, (b) binarized image, (c) smoothed boundary, (d) top left area and bottom left area, (e) turning angle, (f) two reference points, (g) a central point and its ROI, and (h) ROI image. 
The gradient vector points in the direction of the greatest rate of increase of $f(x, y)$. When a palm image is hypothetically considered a $3 \mathrm{D}$ terrain, the intensity value $I(x, y)$ is the height above sea level at a point $(x, y)$. The gradient at a point is a vector that points in the direction of the steepest slope at that point. The steepness of the slope at that point is given by the magnitude of the gradient vector. Thus, the local topographic property in a palm image can be described with the gradient direction and magnitude for each pixel.

The directional feature of the gradient is more robust than the feature of the magnitude with respect to variations in lighting conditions. Thus, this study focuses on the directional feature. To compute a gradient vector, the Kirsch operator [17] is used. The Kirsch operator is a non-linear edge detector that finds the maximum edge strength in a few predetermined directions. The direction of the gradient at point $(x, y)$ is calculated as follows for directions with a $\pi$ 14 difference:

$$
d(x, y)=\arg \max _{k}\left(\sum_{i=-1}^{1} \sum_{j=-1}^{1} g_{i j}^{(k)} I(x+i, y+j)\right), k=0 . .7,
$$

where $g^{(k)}$ is the derivative kernel and $I(x, y)$ is the gray value at point $(x, y)$. The argument $k$ of the maximum value becomes the direction of the gradient vector. Therefore, unlike in Huang's study [18], directional decomposition of a gradient vector is not necessary. This approach is also computationally effective because it requires only integer multiplication and comparison. If an image is crudely convoluted with the derivative kernels, as the edge detector does, the

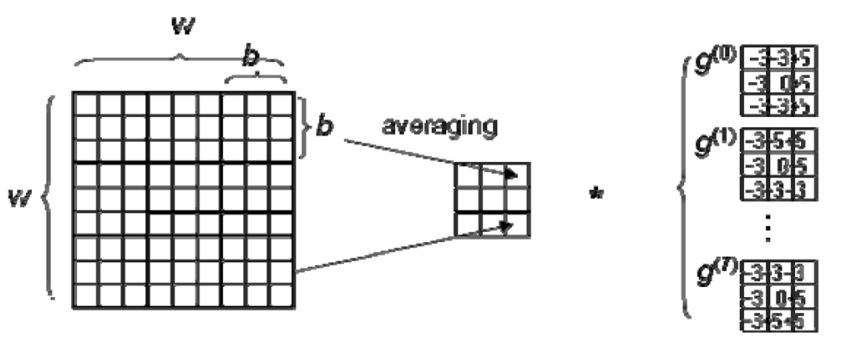

FIG. 2. The convolution operation between a compressed image and derivative kernels. gradient direction is unstable because the $3 \times 3$ area is too small to contain enough information. To overcome this problem, a $w \times w$ scanning window is introduced. The scanning window consists of nine $b \times b$-sized blocks. Fig. 2 shows an example of a $9 \times 9$ scanning window with nine $3 \times 3$ blocks. The local image in the scanning window is compressed into a $3 \times 3$ image by averaging the gray values of the pixels in each block. Then the compressed image is convoluted with each derivative kernel of the Kirsch operator.

\section{FEATURE CODING AND MATCHING}

\subsection{Feature Coding}

Feature coding of the palmprint is performed by replacing all the pixels in the ROI with their index in the gradient direction. Feature coding creates an orientation map. If all the pixels in a cropped ROI image are encoded, if the size of the ROI image is $128 \times 128$, the size of the orientation map is also $128 \times 128$, as shown in Fig. 3 (b). It requires much processing time and creates redundant information. The computation time and the amount of redundant information can be reduced via downsampling. If every fourth pixel is selected, the processing time becomes one-sixteenth shorter, and the size of the orientation map becomes $32 \times 32$. As shown in Fig. 3 (c), feature coding saves most of the important information in the higher-resolution orientation map. On the other hand, if the ROI image is downscaled and convoluted with the same scanning window, orientation maps with different scales can be created. The downscaling is performed by averaging the intensity of the pixels in the non-overlapping $2 \times 2$ window. Fig. 3 (d) and (e) are created from half-scaled and quarter-scaled ROI images, respectively. The orientation map represents local or global gradient information according to the scale.

\subsection{Feature Matching}

The basic strategy for calculating the similarity between two feature images is pixel-to-pixel matching. Let $Q_{o}$ and $R_{O}$ denote the orientation maps that are acquired from a query image $Q$ and a reference image $R$, respectively. Each pixel in $Q_{o}$ is compared with the corresponding pixel in $R_{O}$. The distance between two corresponding pixels in $Q_{O}$ and $R_{O}$ is calculated by modulo distance, because each

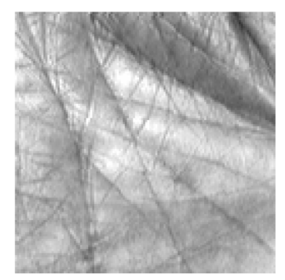

(a)

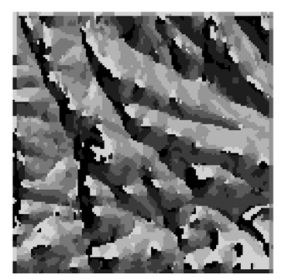

(b)

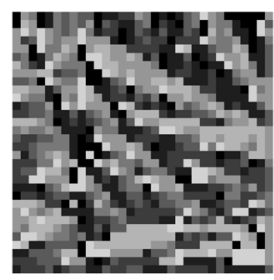

(c)

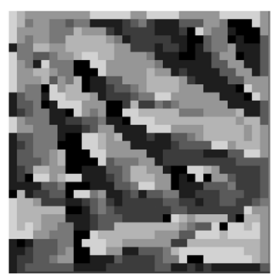

(d)

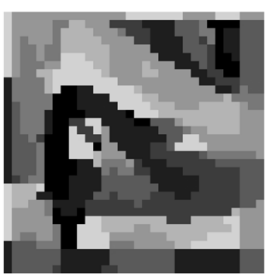

(e)

FIG. 3. An ROI image and its orientation maps: (a) a $128 \times 128$ ROI image, (b) a $128 \times 128$ orientation map, (c) a $32 \times 32$ orientation map, (d) a $32 \times 32$ half-scale orientation map, and (e) a $32 \times 32$ quarter-scale orientation map. 
pixel represents the orientation index between 0 and 7 . The distance of the two images $Q$ and $R$ with the size $m \times n$ can be described as:

$$
d(Q, R)=\frac{\sum_{i=1}^{m} \sum_{j=1}^{n} f_{k}\left(Q_{o}(i, j), R_{O}(i, j)\right) \times\left(Q_{M}(i, j) \wedge R_{M}(i, j)\right)}{\frac{k}{2} \times \sum_{i=1}^{m} \sum_{j=1}^{n}\left(Q_{M}(i, j) \times R_{M}(i, j)\right)},
$$

where the function $f_{k}$ represents the modular $k$ distance (i.e., the distance on the circle: so the modular eight distance between 0 and 7 is 1 , not 7), $Q_{M}$ and $R_{M}$ denote masking maps of $Q$ and $R$; and the symbol ${ }^{\wedge}$ is the logical AND operator. The masking maps $Q_{M}$ and $R_{M}$ are automatically acquired through a thresholding procedure that generates a mask to identify the location of the non-palmprint pixels. If the gray level of a pixel is higher than a predefined threshold, the corresponding pixel at the masking map is 1. Otherwise, the pixel is 0. Masking maps are used to alleviate the mismatch problem caused by the displacement of the palm during the process of data acquisition [15]. Fig. 4 shows examples of two palms captured from the same person, and their ROIs and masking maps.

A translated or rotated palm image is mostly aligned in the preprocessing step, in which the ROI of a palm is extracted. Any remaining variation due to imperfect preprocessing can degrade the performance of the pixel-to-pixel matching method, though. Thus, the pixel-to-cross-shaped area comparison method, which was introduced in the study of Jia et al. [8], was used to improve the matching robustness. The distance function described in Eq. (3) is modified as follows:

$$
d(Q \rightarrow R)=\frac{\sum_{i=1}^{m} \sum_{j=1}^{n} \operatorname{Min}\left(f_{k}\left(Q_{o}(i, j), R_{O}\left(i^{\prime}, j^{\prime}\right)\right)\right) \times\left(Q_{M}(i, j) \wedge R_{M}(i, j)\right)}{\frac{k}{2} \times \sum_{i=1}^{m} \sum_{j=1}^{n}\left(Q_{M}(i, j) \times R_{M}(i, j)\right)},
$$

where $\left(i, j^{\prime}\right) \in\{(i-1, \mathrm{j}),(i, j-1),(i, j),(i, j+1),(i+1, j)\}$. The function $d(Q \rightarrow R)$ represents the distance from $Q$ to $R$. The distance from $R$ to $Q$ can also be described as $d(R \rightarrow$ $Q$ ). Finally, the distance function can be calculated as follows.

$$
d(Q \leftrightarrow R)=\min (d(Q \rightarrow R), d(R \rightarrow Q))
$$
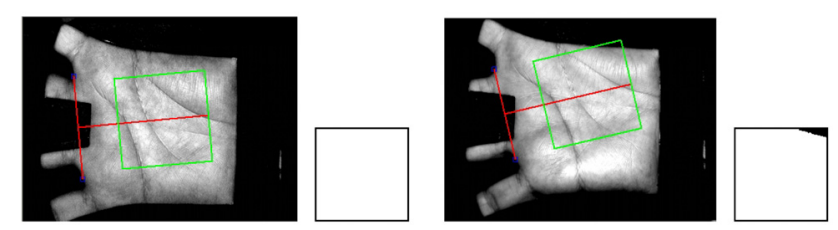

FIG. 4. Palm images captured from the same person: (a) example of the normal placement of a palm and its ROI and masking map, and (b) example of the misplacement of a palm and its ROI and masking map.

\subsection{Fusion of Matching Distances}

In this study, three orientation maps were made with different scales to represent local and global gradient information. The local gradient information is extracted from the cropped $128 \times 128$ ROI images, and its two downscaled $64 \times 64$ and $32 \times 32$ images are used to represent the gradient information in the wider area. Before the matching distances that were obtained from the multi-scale orientation maps were fused, distance normalization was required because they have different statistical properties such as their mean, deviation, and minimum and maximum values. The Min-Max method described in Eq. (6) was used for the normalization. The quantities $\max (D)$ and $\min (D)$ specify the end points of the distance range. This method maps the raw distances to the $[0,1]$ range.

$$
n=\frac{d-\min (D)}{\max (D)-\min (D)}
$$

Two or more normalized distances can be fused together to get the final matching distance. The final matching distance between a query image $Q$ and a reference image $R$ is denoted by the following equation:

$$
d_{f}(Q \leftrightarrow R)=\sum_{m=1}^{M} W_{m} n_{m},
$$

where $w_{m}$ and $n_{m}$ denote the weight and the normalized distance of the matcher $\mathrm{m}$, respectively, and $M$ is the number of matchers. The weight, $w_{m}$, is determined according to each fusion method.

\section{EXPERIMENTAL RESULTS}

\subsection{Palmprint Database}

The widely used public database, PolyU [19], was used to evaluate the performance of the proposed method. The database contains a total of 7,752 images from 386 different palms. The palmprint images were collected in two sessions. In each of the sessions, around 10 samples for each palm were captured. The average interval between the first and second sessions was about two months. In addition, the light source and the focus of the CCD camera were changed so that the images that were collected in the first and second sessions could be regarded as having been captured by two different devices [8]. An LED was used in the first session, and an incandescent lamp was used in the second session. Also, the lenses in the two sessions slightly differed, and the focus in the first session was slightly longer. Some samples in this database are shown in Fig. 5, in which the four samples in the top row were captured in the first session and those in the bottom row were captured in the second session. The two images in the same column were 


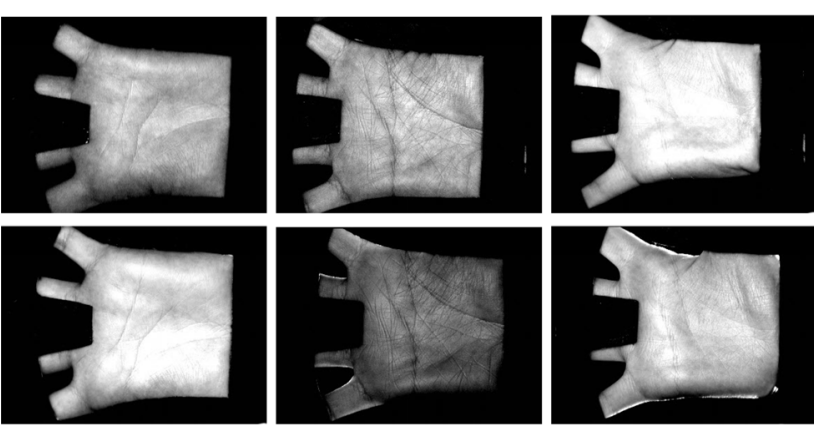

FIG. 5. Some samples from the PolyU palmprint database.

captured from the same palm at different sessions.

The database was divided into two data sets: training and testing. The training and testing data sets consisted of the samples that were taken in the first and second sessions, respectively. Only the training data set was used to adjust the parameters of the size of the scanning window and the resolution of the orientation map. Palmprint verification was performed by matching a sample taken from the testing data set with the registered templates that were made from the training data set. This is a more realistic experiment because the two data sets were not collected in the same session, as is always the case in real-world applications.

\subsection{Selection of the Window Size and the Resolution}

To test the effects of some parameters, 1,000 samples were selected from 200 different palms in the training dataset. Only the first sample of each palm was selected to create the reference model, and the other four samples of each palm were used for testing. The size of the scanning window and the resolution of the orientation map are important parameters that affect the recognition accuracy and the processing time. The size of the scanning window controls the sensitivity of the local intensity variation, and the shifting unit of the scanning window controls the resolution of the orientation map. If the shifting unit doubles, the resolution of the orientation map is halved both horizontally and vertically. The nearest neighbour classifier was used for the recognition. Fig. 6 shows the recognition rate for the different sizes of the scanning window with different resolutions of the orientation map. When the local image in the scanning window was directly convoluted with the derivative kernels, that is, when the $3 \times 3$ scanning window was used, poor results were achieved. In particular, when the size of the orientation map was $32 \times 32$ or less, the performance was drastically degraded. In the case when the compressed image was used, promising results were achieved. In addition, the performance was well-preserved even in the $32 \times 32$ orientation map. The larger the scanning window and the higher the resolution of the orientation map were, the more processing time was needed, so the $9 \times 9$ scanning window and the $32 \times 32$-resolution orientation map were used.

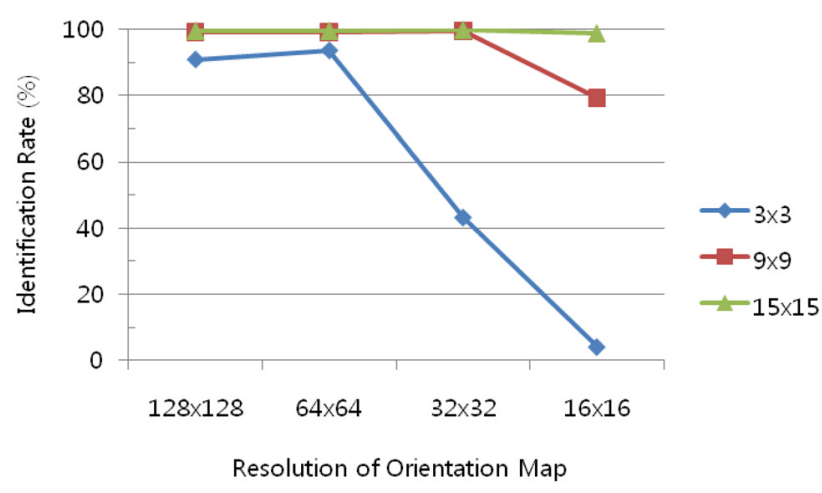

FIG. 6. The effects of the size of the scanning window and the resolution of the orientation map.

\subsection{Palmprint Verification}

A biometric system classifies an individual as either a genuine user or an impostor. Thus, the system may commit two types of recognition error: it may either falsely accept an impostor or falsely reject a genuine user. If the matching distance does not exceed the appointed threshold, the palmprint is accepted. Otherwise, it is rejected. The false acceptance rate (FAR) is the probability that an unauthorized individual will be accepted, and the false rejection rate (FRR) is the probability that an authorized user will be inappropriately rejected. If a system is designed so that it would be more difficult for an impostor to enter by adjusting the threshold (i.e., reducing the FAR), the system also becomes more difficult for a valid person to enter (i.e., the FRR will increase) [20]. Thus, the two rates contradict each other and cannot be lowered at the same time. The genuine acceptance rate (GAR) is also dependent on the threshold, because it is calculated with 1-FRR at a specific FAR. The equal error rate (EER) is independent of the threshold, however, because it is the rate at which the FAR is equal to the FRR. Therefore, the EER can be used as an application-independent metric. The decidability index $d^{\prime}$ [21] does not use the threshold but the statistics of two groups: the genuine group and the impostor group. Thus, it is also an applicationindependent metric. For this reason, the EER and the decidability index $d$ ' were used to evaluate the performance of the proposed method.

Three data sets, $N=100,200$, and 386 , were used to evaluate the verification accuracy and extensibility of the proposed method. Each set contained all the samples of $N$ different palms in the testing data set. When $N=386$, the total number of matchings was about $1,489,960(386 \times 10 \times 386)$ for each scale. The number of genuine matches was 3,860 $(386 \times 10)$, and the rest were impostor matches. Table 1 shows the results of the matching with the three data sets. Generally, the downsampled $64 \times 64$ images performed best with respect to the EER, and the original $128 \times 128 \mathrm{ROI}$ images performed best with respect to the index $d$ '. As the number of subjects increases, the EER rises and the index $d$ ' falls. The decreasing rate of performance is less, however, 
TABLE 1. Comparison of the verification results with different scales and data sets

\begin{tabular}{c|c|c|c|c|c|c}
\hline \multirow{2}{*}{ ROI Size } & \multicolumn{2}{|c|}{$128 \times 128$} & \multicolumn{2}{c}{$64 \times 64$} & \multicolumn{2}{c}{$32 \times 32$} \\
\cline { 2 - 7 } & EER $(\%)$ & $d^{\prime}$ & EER $(\%)$ & $d^{\prime}$ & EER (\%) & $d^{\prime}$ \\
\hline Set 1 $(N=100)$ & 0.737 & 5.163 & 0.982 & 5.073 & 3.929 & 3.654 \\
\hline Set 2 $(N=200)$ & 1.461 & 4.998 & 1.371 & 4.942 & 4.119 & 3.583 \\
\hline Set 3 $(N=386)$ & 1.796 & 4.802 & 1.435 & 4.795 & 4.200 & 3.519 \\
\hline
\end{tabular}

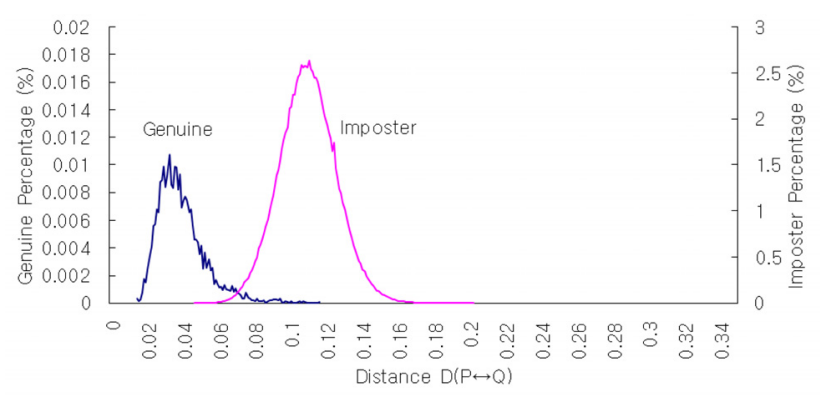

(a) The results of the $128 \times 128 \mathrm{ROI}$ images

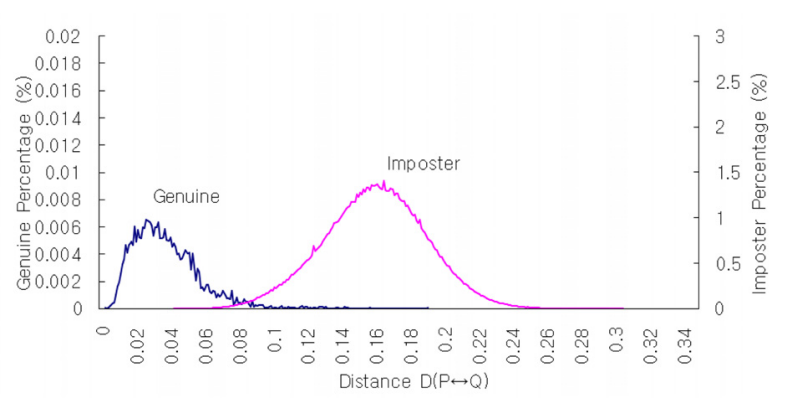

(b) The results of the $64 \times 64$ ROI images

FIG. 7. The distributions of the genuine and impostor matching distances $(N=386)$.

TABLE 2. Comparison of the verification results of four different fusion methods

\begin{tabular}{c|c|c|c|c|c|c|c|c}
\hline \multirow{2}{*}{ Matcher Fusion Method } & \multicolumn{2}{|c|}{ Simple-Sum } & \multicolumn{2}{c|}{ Min-Score } & \multicolumn{2}{c|}{ Max-Score } & \multicolumn{2}{c}{ Matcher Weighting } \\
\cline { 2 - 21 } & EER (\%) & $d^{\prime}$ & EER (\%) & $d^{\prime}$ & EER (\%) & $d^{\prime}$ & EER (\%) & $d^{\prime}$ \\
\hline $\mathrm{M}_{1}+\mathrm{M}_{2}$ & 0.580 & 6.090 & 0.614 & 5.706 & 0.675 & 6.215 & 0.560 & 6.078 \\
\hline $\mathrm{M}_{1}+\mathrm{M}_{2}+\mathrm{M}_{3}$ & 0.546 & 5.844 & 0.572 & 4.770 & 0.800 & 5.966 & 0.515 & 5.934 \\
\hline
\end{tabular}

than the increasing rate of $N$. This means that the proposed method is extensible to a larger dataset. The distribution of the genuine and impostor matching distances when $N=$ 386 is shown in Fig. 7. There are two distinct peaks in the distributions. One corresponds to the correct matching distance, and the other corresponds to the incorrect matching distance. These two peaks are widely separated, and the overlapping area is very small. The distributions differ with the scale of the ROI images. The distributions of the downscaled images are more broadly spread than those of the original ROI images.

To find the fusion method that can enhance the accuracy of the palmprint verification, four different fusion methods were experimented on: simple-sum, min-score, max-score, and matcher weighting [22]. Table 2 shows the verification results of the fusion methods, where $M_{1}, M_{2}$, and $M_{3}$ denote the matchers that used the features taken from the $128 \times 128,64 \times 64$, and $32 \times 32$ ROI images, which is acquired by downscaling with $1 / 1,1 / 2,1 / 4$, respectively. Three different downsampling rates of $1 / 4,1 / 2,1 / 1$ were used to get the gradient orientation maps of $32 \times 32$. In consequence, it produces the orientation map of the same resolution regardless of the different sizes of ROI images. All the fusion methods improved the verification performance. In particular, the matcher-weight fusion method showed the greatest performance improvement. The effect on the performance improvement with the addition of one additional scale to the two-scale orientation map was not significant.

\subsection{Comparison with Other Palmprint Verification Methods}

The approach in this study was compared with three line-based methods that are based on orientation coding: competitive coding (CompCode), palmprint orientation coding (POC), and robust line orientation coding (RLOC). To compare the features of these different methods under the same conditions, the preprocessing routine was shared and the same strategy for pixel-to-cross-shaped area matching was used. The verification results are described in Table 3.

Comparison of the four methods in which a single-scale feature was used showed that the proposed approach $M_{2}$ had the lowest EER and that CompCode had the highest $d$ '. The fused method, however, in which orientation maps with three different scales were used, performed best not only in terms of the EER but also in terms of the decidability $(d$ '). These results validate the effectiveness of the proposed gradient orientation feature. It also shows that the verification could be greatly improved by fusing orientation maps with different scales. 
TABLE 3. Comparison of the verification results of the line-based methods

\begin{tabular}{c|c|c|c|c|c}
\hline \hline Method Performance & Comp-Code & POC & RLOC & $\mathrm{M}_{2}$ & $\mathrm{M}_{1}+\mathrm{M}_{2}+\mathrm{M}_{3}$ \\
\hline EER (\%) & 2.093 & 2.436 & 3.000 & 1.435 & 0.515 \\
\hline$d^{\prime}$ & 5.060 & 4.185 & 4.261 & 4.795 & 5.934 \\
\hline
\end{tabular}

\section{CONCLUSIONS}

In this study, a new approach to palmprint verification based on gradients was proposed. A palm image was hypothetically considered a 3D terrain. From this viewpoint, the principal lines and wrinkles become deep and shallow valleys. Most previous studies based on orientation coding focused on the direction of the valley itself, but this study focused on the steepest slope direction in each local area, where the slope is mainly caused by the valleys.

The experimental results showed that the gradient orientation feature that was obtained using the Kirsch operator is highly effective in palmprint verification. They also showed that the proposed method is superior to other methods based on line-based orientation coding. In particular, the fusion of orientation maps with different scales greatly improved the verification, regardless of the fusion method. Although this work was presented in the context of palmprint verification, the proposed approach is general enough, and the ideas can be applied to other biometrics such as iris and finger knuckle verification.

\section{REFERENCES}

1. D. Zhang, X. Jing, and J. Yang, Biometric Image Discrimination Technologies (Idea Group Publishing, USA, 2006), Chapter 1.

2. B. Kang and K. Park, "Multimodal biometric authentication based on the fusion of finger vein and finger geometry," Opt. Eng. 48, 090501 (2009).

3. M. Jeong, "Analysis of fingerprint recognition characteristics based on new CGH direct comparison method and nonlinear joint transform correlator," J. Opt. Soc. Korea 13, 445-450 (2009).

4. A. Kumar, D. Wong, H. Shen, and A. Jain, "Personal verification using palmprint and hand geometry biometric," in Proc. The 4th AVBPA (Guilford, UK, June 2003), LNCS 2688, pp. 668-678.

5. A. Kong and D. Zhang, "Competitive coding scheme for palmprint verification," in Proc. The 17th ICPR (Cambridge, UK, August 2004), pp. 520-523.

6. F. Yue, W. Zuo, D. Zhang, and K. Wang, "Competitive code-based fast palmprint identification using a set of cover trees," Opt. Eng. 48, 067204 (2009).

7. X. Wu, K. Wang, and D. Zhang, "Palmprint authentication based on orientation code matching," in Proc. The 5th $A V B P A$ (New York, USA, July 2005), LNCS 3546, pp. 555-562.

8. W. Jia, D. Huang, and D. Zhang, "Palmprint verification based on robust line orientation code," Pattern Recognition 41, 1504-1513 (2008).

9. G. Lu, D. Zhang, and K. Wang, "Palmprint recognition using eigenpalms features," Pattern Recognition Letters 24, 1463-1467 (2003).

10. M. Ekinci and M. Aykut, "Gabor-based kernel PCA for palmprint recognition,” Electron. Lett. 43, 1077-1079 (2007).

11. X. Wu, D. Zhang, and K. Wang, "Fisherpalms based palmprint recognition," Pattern Recognition Letters 24, 2829-2838 (2003).

12. G. Lu, K. Wang, and D. Zhang, "Wavelet based independent component analysis for palmprint recognition," in Proc. The 3rd ICMLC (Alberta, Canada, July 2004), pp. 3547-3550.

13. Y. Han, T. Tan, and Z. Sun, "Palmprint recognition based on directional features and graph matching," in Proc. The 2nd ICB (Seoul, Korea, August 2007), LNCS 4642, pp. 1164-1173.

14. X. Pan and Q. Ruan, "Palmprint recognition using Gaborbased local invariant features," Neurocomputing 72, 2040-2045 (2009).

15. D. Zhang, W. Kong, J. You, and M. Wong, "Online palmprint identification," IEEE Transactions on PAMI 25, 1041-1050 (2003).

16. V. Struc and N. Pavesic, "Phase congruency features for palm-print verification," IET Signal Processing 3, 258-268 (2009).

17. R. Kirsch, "Computer determination of the constituent structure of biological images," Computers \& Biomedical Research 4, 315-328 (1971).

18. L. Huang, A. Shimizu, Y. Hagihara, and H. Kobatake, "Gradient feature extraction for classification-based face detection," Pattern Recognition 36, 2501-1511 (2003).

19. PolyU Palmprint Database, available at http://www4.comp. polyu.edu.hk/ biometrics/.

20. $\mathrm{X}$. Wu, K. Wang, and D. Zhang, "Wavelet energy feature extraction and matching for palmprint recognition," Journal of Computer Science and Technology 20, 411-418 (2005).

21. J. Daugman, "The importance of being random: statistical principles of iris recognition," Pattern Recognition 36, 279-291 (2003).

22. R. Snelick, U. Uludag, A. Mink, M. Indovia, and A. Jain, "Large-scale evaluation of multimodal biometric authentication using state-of-the-art systems," IEEE Transactions on PAMI 27, 450-455 (2005). 